\title{
AN ANALYSIS OF THE MODIFIED L1 SCHEME FOR TIME-FRACTIONAL PARTIAL DIFFERENTIAL EQUATIONS WITH NONSMOOTH DATA
}

\author{
YUBIN YAN, MONZORUL KHAN, AND NEVILLE J. FORD *
}

\begin{abstract}
We introduce a modified L1 scheme for solving time fractional partial differential equations and obtain error estimates for smooth and nonsmooth initial data in both homogeneous and inhomogeneous cases. Jin et al. (2016, An analysis of the L1 scheme for the subdiffusion equation with nonsmooth data, IMA J. of Numer. Anal., 36, 197-221) established an $O(k)$ convergence rate for the L1 scheme for smooth and nonsmooth initial data for the homogeneous problem, where $k$ denotes the time step size. We show that the modified L1 scheme has convergence rate $O\left(k^{2-\alpha}\right), 0<\alpha<1$ for smooth and nonsmooth initial data in both homogeneous and inhomogeneous cases. Numerical examples are given to show that the numerical results are consistent with the theoretical results.
\end{abstract}

Key words. time fractional partial differential equations, Caputo fractional derivative, error estimates, Laplace transform

AMS subject classifications. 26A33, 65M06, 65M12, 65M15, 35R11

1. Introduction. Consider the following time fractional partial differential equation, with $0<\alpha<1$,

$$
{ }_{0}^{C} D_{t}^{\alpha} u(t)+A u(t)=f(t), \quad \text { for } 0<t \leq T, \quad \text { with } u(0)=u_{0},
$$

where ${ }_{0}^{C} D_{t}^{\alpha} u(t)$ denotes the Caputo fractional derivative defined by

$$
{ }_{0}^{C} D_{t}^{\alpha} u(t)=\frac{1}{\Gamma(1-\alpha)} \int_{0}^{t}(t-s)^{-\alpha} u^{\prime}(s) d s,
$$

and $u^{\prime}(s)=\partial u / \partial s$ and $A$ is a selfadjoint positive definite second order elliptic partial differential operator in a bounded regular domain $\Omega \subset \mathbb{R}^{d}, d=1,2,3$, with $\mathcal{D}(A)=$ $H_{0}^{1}(\Omega) \cap H^{2}(\Omega)$, where $H_{0}^{1}(\Omega), H^{2}(\Omega)$ denote the standard Sobolev spaces. We also denote $L_{2}(\Omega)$ the standard square integrable function space with norm $\|\cdot\|$.

The equation (1.1) can be written as, [9]

$$
{ }_{0}^{R} D_{t}^{\alpha}(u(t)-u(0))+A u(t)=f(t), \quad \text { for } 0<t \leq T,
$$

where ${ }_{0}^{R} D_{t}^{\alpha} u(t)$ denotes the Riemann-Liouville fractional derivative defined by

$$
{ }_{0}^{R} D_{t}^{\alpha} u(t)=\frac{1}{\Gamma(1-\alpha)} \frac{d}{d t} \int_{0}^{t}(t-s)^{-\alpha} u(s) d s .
$$

Our analysis will use Laplace transform method. The assumption that $A$ is positive definite implies that $A$ generates an analytic semigroup, so that for some $\pi / 2<\theta_{0}<\pi$ and with $C=C_{\theta_{0}}$ we have the resolvent estimate, see Lubich et al. [30], Thomée [38],

$$
\left\|(z I+A)^{-1}\right\| \leq C|z|^{-1}, \quad \text { for } z \in \Sigma_{\theta_{0}}=\left\{z \neq 0:|\arg z|<\theta_{0}\right\} .
$$

* Yubin Yan: Department of Mathematics, University of Chester, Thornton Science Park, Pool Lane, Ince, CH2 4NU, UK, (y.yan@chester.ac.uk), Monzorul Khan: Department of Mathematics, University of Chester, Thornton Science Park, Pool Lane, Ince, CH2 4NU, UK, (sohel_ban@yahoo.com), Neville J. Ford: Department of Mathematics, University of Chester, Thornton Science Park, Pool Lane, Ince, CH2 4NU, UK, (njford@chester.ac.uk). Dr. Yubin Yan is the corresponding author. 
In our analysis, we will choose $\theta>\pi / 2$ close to $\pi / 2$ such that $\theta<\theta_{0}$ which implies that $z^{\alpha} \in \Sigma_{\theta_{0}}$ for any $z \in \Sigma_{\theta}$ since $\arg \left(z^{\alpha}\right)=\alpha \theta<\theta<\theta_{0}$ for $0<\alpha<1$. Hence there exists a constant $C$ which depends only on $\theta$ and $\alpha$ such that, see Jin et al. [20, (2.3)],

$$
\left\|\left(z^{\alpha} I+A\right)^{-1}\right\| \leq C|z|^{-\alpha}, \quad \forall z \in \Sigma_{\theta}=\{z \neq 0:|\arg z|<\theta\} .
$$

Further we choose $\theta>\pi / 2$ close to $\pi / 2$ such that $z_{k}^{\alpha} \in \Sigma_{\theta_{0}}$ for $z \in \Gamma$ which implies that $\left(z_{k}^{\alpha} I+A\right)^{-1}$ exists where $z_{k}$ is defined in (2.5) and $\Gamma=\Gamma_{\theta}=\{z:|\arg z|=\theta\}$.

Many application problems can be modelled by (1.1), for example, thermaldiffusion in media with fractional geometry [35], highly heterogeneous aquifer [1], underground environmental problems [18], random walks [17], [31], etc.

There has been much recent interest in developing numerical methods for (1.1), especially spectral methods, [4], [5], [43], [45], and the discontinuous Galerkin method [8], [32], [33], [34]. In this paper, we will consider some time discretization schemes for (1.1) using the direct approximation of the time fractional derivative. There are two predominant approaches for approximating the fractional derivative: one approach is by using Lubich's convolution quadrature [27]-[29] and another approach is by using the L1 scheme (or Diethelm's finite difference method). For the recent developments for solving fractional ordinary (or partial ) differential equations by using the Lubich's convolution quadrature method, readers may refer to e.g., [39], [11], [3], [42], [6], [44], [46], [47], [22], [21], [19], etc.

Let us briefly review the approach for approximating the fractional derivative by using the L1 scheme (or Diethelm's finite difference method) which we will forcus on in this paper. The L1 scheme may be obtained by the direct approximation of the derivative in the definition of the Caputo fractional derivative, e.g., [25], [24], [16], [26], [37], or by the approximation of the Hadamard finite-part integral, e.g., [9], [10], [13], [14], [15], [23], [41]. Since its first appearance the L1 scheme has been extensively used in practice and currently it is one of the most popular and successful numerical methods for solving the time fractional diffusion equation.

Recently, Jin et al. [20] obtained the error estimates of the L1 scheme for solving (1.1) with the convergence order $O(k)$ for smooth and nonsmooth initial data in the homogeneous case, i.e., $f=0$. We will introduce a modified L1 scheme for solving (1.1) and prove that this scheme has the optimal convergence order $O\left(k^{2-\alpha}\right)$ in both homogeneous and inhomogeneous cases for smooth and nonsmooth initial data. Our error estimates depend only on data regularity, without assuming any compatibility conditions on the source term. We derive the error estimates by using the techniques developed in Lubich et al. [30] for solving the integro-differential equation, see also $[36],[7],[2]$. We shall use some delicate estimates of the kernel function which involves the polylogarithmic functions, see Jin et al. [20].

Let $u(t)-u_{0}=V(t)$. Then (1.1) is equivalent to, with $u_{0} \in \mathcal{D}(A)$,

$$
{ }_{0}^{C} D_{t}^{\alpha} V(t)+A V(t)=-A u_{0}+f(t), 0<t \leq T, \quad \text { with } V(0)=0 .
$$

It proves more convenient to consider the error estimates of the time discretization scheme for solving (1.5) instead of solving (1.1), see [30].

The homogeneous equation of (1.5) reads, with $u_{0} \in \mathcal{D}(A)$,

$$
{ }_{0}^{C} D_{t}^{\alpha} V(t)+A V(t)=-A u_{0}, \quad \text { with } V(0)=0 .
$$

Let $0=t_{0}<t_{1}<\cdots<t_{N}=T$ be a partition of $[0, T]$ and $k$ the time step size. Let $V^{n} \approx V\left(t_{n}\right), n=0,1,2, \ldots, N$ be the approximate solutions of $V\left(t_{n}\right)$. We first 
define the following time discretization scheme for solving the homogeneous equation (1.6), with $u_{0} \in \mathcal{D}(A)$,

$$
k^{-\alpha} \sum_{j=1}^{n} w_{n-j} V^{j}+A V^{n}=-A u_{0}, n \geq 1, \quad \text { with } V^{0}=0 .
$$

where the weights $w_{j}, j=1,2, \ldots, n, n \geq 1$ are defined by (2.4).

Jin et al. [20, Theorem 3.16] proved the following nonsmooth data error estimates:

Theorem 1.1. ([20, Theorem 3.16]) Let $V\left(t_{n}\right)$ and $V^{n}$ be the solutions of (1.6) and (1.7), respectively. Let $u_{0} \in L_{2}(\Omega)$. Then we have, with $0<\alpha<1$,

$$
\left\|V\left(t_{n}\right)-V^{n}\right\| \leq C k t_{n}^{-1}\left\|u_{0}\right\|, n \geq 1
$$

REMARK 1.2. In the time discretization scheme (1.7), we require $A u_{0} \in L_{2}(\Omega)$, i.e., the initial data $u_{0}$ is reasonably smooth. However one may use the scheme (1.7) to prove the error estimates with the nonsmooth initial data $u_{0} \in L_{2}(\Omega)$. This idea has been used in Lubich et al. [30, (1.8)] and Jin et al. [22, Remark 2.4]. The similar remark is also for our modified L1 scheme (1.9)-(1.11) below.

To improve the convergence rate of the L1 scheme (1.7) for solving (1.6), we introduce the following modified L1 scheme: with $c_{0}=1 / 2$, with $u_{0} \in \mathcal{D}(A)$,

$$
\begin{aligned}
& k^{-\alpha} \sum_{j=1}^{n} w_{n-j} V^{j}+A V^{n}=\left(-A u_{0}\right)\left(1+c_{0}\right), \text { for } n=1, \\
& k^{-\alpha} \sum_{j=1}^{n} w_{n-j} V^{j}+A V^{n}=-A u_{0}, \text { for } n \geq 2, \\
& V^{0}=0
\end{aligned}
$$

where the weights $w_{n-j}, j=1,2, \ldots, n$ are given by (2.4). We then have the following nonsmooth data error estimates:

THEOREM 1.3. Let $V\left(t_{n}\right)$ and $V^{n}$ be the solutions of (1.6) and (1.9)-(1.11), respectively. Let $u_{0} \in L_{2}(\Omega)$. We have

$$
\left\|V\left(t_{n}\right)-V^{n}\right\| \leq C k^{2-\alpha} t_{n}^{\alpha-2}\left\|u_{0}\right\| .
$$

Based on the modified L1 scheme (1.9)-(1.11), we introduce the following modified L1 scheme for solving the inhomogeneous equation (1.5), with $V^{0}=0$ and $u_{0} \in \mathcal{D}(A)$,

$$
\begin{aligned}
& k^{-\alpha} \sum_{j=1}^{n} w_{n-j} V^{j}+A V^{n}=-A u_{0}+f\left(t_{n}\right)+c_{0}\left(-A u_{0}+f(0)\right), n=1, \\
& k^{-\alpha} \sum_{j=1}^{n} w_{n-j} V^{j}+A V^{n}=-A u_{0}+f\left(t_{n}\right), n=2,3, \ldots, N,
\end{aligned}
$$

where $w_{j}, j=0,1,2, \ldots$ are defined by $(2.4)$.

We obtain the following error estimates with nonsmooth data: 
TheOREM 1.4. Let $V\left(t_{n}\right)$ and $V^{n}$ be the solutions of (1.5) and (1.12)-(1.13), respectively. Let $u_{0} \in L_{2}(\Omega)$. Then we have, with $0<\alpha<1$,

$\left\|V\left(t_{n}\right)-V^{n}\right\| \leq C k^{2-\alpha}\left(t_{n}^{\alpha-2}\left\|u_{0}\right\|+t_{n}^{2 \alpha-2}\|f(0)\|+t_{n}^{2 \alpha-1}\left\|f^{\prime}(0)\right\|+\int_{0}^{t_{n}}\left(t_{n}-s\right)^{2 \alpha-1}\left\|f^{\prime \prime}(s)\right\| d s\right)$.

The main contributions of this paper are as follows:

- we introduce the modified L1 scheme for solving time-fractional partial differential equations and prove that the convergence rate of this scheme is $O\left(k^{2-\alpha}\right), 0<\alpha<1$ for both smooth and nonsmooth initial data in the homogeneous case.

- we also obtain error estimates of the modified L1 scheme in the inhomogeneous case for smooth and nonsmooth initial data.

The rest of the paper is organized as follows. In Section 2, we consider the error estimates for the homogeneous problem and in Section 3, we consider the error estimates for the inhomogeneous problem. Numerical examples are given in Section 4.

Throughout, the notations $C$ and $c$, with or without a subscript, denote generic constants, which may differ at different occurrences, but are always independent of the step size $k$.

2. The homogeneous problem. In this section we will consider the time discretization scheme for solving the homogeneous equation (1.5).

Recall that the Caputo fractional derivative can be approximated by using the so-called L1 scheme, see [20],

${ }_{0}^{C} D_{t}^{\alpha} V\left(t_{n}\right)=k^{-\alpha}\left(b_{0} V\left(t_{n}\right)+\sum_{j=1}^{n-1}\left(b_{j}-b_{j-1}\right) V\left(t_{n-j}\right)-b_{n-1} V(0)\right)+O\left(k^{2-\alpha}\right), k \rightarrow 0$,

where the weights $b_{j}$ are given by

$$
b_{j}=\left((j+1)^{1-\alpha}-j^{1-\alpha}\right) / \Gamma(2-\alpha), j=0,1,2, \ldots, n-1 .
$$

Rearranging the coefficients, we may write

$$
{ }_{0}^{C} D_{t}^{\alpha} V\left(t_{n}\right)=k^{-\alpha} \sum_{j=0}^{n} w_{n-j, n} V\left(t_{j}\right)+O\left(k^{2-\alpha}\right), k \rightarrow 0,
$$

where $w_{j, n}, j=0,1,2, \ldots, n$ are given by

$$
\Gamma(2-\alpha) w_{j, n}=\left\{\begin{array}{l}
1, \quad \text { for } j=0, \\
-2 j^{1-\alpha}+(j-1)^{1-\alpha}+(j+1)^{1-\alpha}, \text { for } j=1,2, \ldots, n-1, \\
(j-1)^{1-\alpha}-j^{1-\alpha}, \text { for } j=n .
\end{array}\right.
$$

We remark that the above weights $w_{j, n}, j=0,1,2, \ldots, n$ can also be obtained by using Diethelm's finite difference method [9]. More precisely, the L1 scheme for approximating the Caputo fractional derivative may be obtained first by approximating the Riemann-Liouville fractional derivative with Diethelm's finite difference method [9] and then applying the relation between the Riemann-Liouville and Caputo fractional derivatives, i.e., ${ }_{0}^{C} D_{t}^{\alpha} V(t)={ }_{0}^{R} D_{t}^{\alpha}(V(t)-V(0))$ for $0<\alpha<1$. (In our case $V(0)=0)$. 
2.1. L1 scheme. We now define the following L1 scheme for solving (1.6),

$$
k^{-\alpha} \sum_{j=0}^{n} w_{n-j, n} V^{j}+A V^{n}=-A u_{0}, n \geq 1, \quad \text { with } V^{0}=0,
$$

or

$$
k^{-\alpha} \sum_{j=1}^{n} w_{n-j, n} V^{j}+A V^{n}=-A u_{0}, n \geq 1, \quad \text { with } V^{0}=0 .
$$

For any fixed $n \geq 1$, we observe that $w_{j, n}, j=0,1, \ldots, n-1$ only depend on $j=0,1,2, \ldots, n-1$. For example, we have $w_{0, n}=1 / \Gamma(2-\alpha)$ for any $n \geq 1$, $w_{1, n}=1 / \Gamma(2-\alpha)\left((-2) 1^{1-\alpha}+(1-1)^{1-\alpha}+(1+1)^{1-\alpha}\right)$ for any $n \geq 2, \ldots$ Therefore, we may write $w_{0}=w_{0, n}, w_{1}=w_{1, n}, w_{2}=w_{2, n}, \ldots, w_{n-1}=w_{n-1, n}$ for any fixed $n \geq 1$. More precisely, we define $w_{j}, j=0,1,2, \ldots$ as follows

$$
\Gamma(2-\alpha) w_{j}=\left\{\begin{array}{l}
1, \quad \text { for } j=0, \\
-2 j^{1-\alpha}+(j-1)^{1-\alpha}+(j+1)^{1-\alpha}, \text { for } j=1,2, \ldots
\end{array}\right.
$$

Our time discretization scheme (1.7) in the introduction section is then defined by using the weights $w_{j}, j=0,1,2, \ldots$ in $(2.4)$.

We remark that in the proof of the error estimates below, we shall see that it is necessary to use the notations $w_{j}, j=0,1,2, \ldots$ in (1.7) instead of using the notations $w_{n-j, n}$ in (2.3) since we need to apply the discrete Laplace transform of the sequence $\left(w_{0}, w_{1}, w_{2}, \ldots\right)$.

The error estimate in Theorem 1.1 was proved in Jin et al. [20, Theorem 3.16]. For completeness, we will give the idea of the proof of Theorem 1.1 in a slightly simpler way in the next subsections. We then follow the same idea to prove the error estimates for the modified L1 scheme later.

2.1.1. Some lemmas. To prove Theorem 1.1, we need to show that $z_{k}^{\alpha} \in \Sigma_{\theta_{0}}$ for some $\theta_{0} \in(\pi / 2, \pi)$ where $z_{k}$ is defined in (2.5) below and $\theta_{0}$ is introduced in (1.3).

Lemma 2.1. [20, Lemma 3.7] Let $\theta>\pi / 2$ be close to $\pi / 2$. Let $z \in \Gamma_{k}$ with $\Gamma_{k}=\{z \in \Gamma:|\Im z| \leq \pi / k\}$ and $\Gamma=\{z:|\arg z|=\theta\}$ (with $\Im z$ running from $-\infty$ to $\infty)$. Denote

$$
z_{k}=\frac{\delta(\zeta)}{k}, \quad \text { with } \delta(\zeta)^{\alpha}=\sum_{j=0}^{\infty} w_{j} \zeta^{j}, \quad \zeta=e^{-z k}
$$

where $w_{j}, j=0,1,2, \ldots$ are defined by (2.4). Then there exists $\theta_{0} \in(\pi / 2, \pi)$ such that

$$
z_{k}^{\alpha} \in \Sigma_{\theta_{0}}, \quad \text { for all } z \in \Sigma_{\theta}
$$

REMARK 2.2. In Lemma 3.7 in Jin et al. [20], the authors proved that for all $\pi / 2<\theta<\pi$, there exists $\theta_{0} \in(\pi / 2, \pi)$ such that $z_{k}^{\alpha} \in \Sigma_{\theta_{0}}$ for all $z \in \Sigma_{\theta}$. Actually in our analysis, we only need to show $z_{k}^{\alpha} \in \Sigma_{\theta_{0}}$ for all $z \in \Sigma_{\theta}$ for some $\theta>\pi / 2$ close to $\pi / 2$. 
We also need the following lemmas in the proof of Theorem 1.1.

Lemma 2.3. Let $w_{j}, j=0,1,2, \ldots$ be defined by (2.4). We have the following singularity expansion, with $\zeta=e^{-z k}$,

$$
\sum_{j=0}^{\infty} w_{j} \zeta^{j}=(z k)^{\alpha}+c_{2}(z k)^{2}+c_{3}(z k)^{3}+\ldots
$$

for some suitable constants $c_{2}, c_{3}, \ldots$.

To prove Lemma 2.3, we need to introduce the polylogorithm function

$$
\operatorname{Li}_{p}(z)=\sum_{j=1}^{\infty} \frac{z^{j}}{j^{p}}
$$

The polynomial function $\operatorname{Li}_{p}(z)$ is well defined for $|z|<1$ and $p \in \mathbb{C}$. It can be analytically continued to the split complex plane $\mathbb{C} \backslash[1,+\infty)$; see Flajolet [12]. With $z=1$, it recovers the Riemann zeta function $\varsigma(p)=\mathrm{Li}_{p}(1)$. We also recall an important singular expansion of the function $\operatorname{Li}_{p}\left(e^{-z}\right)$ (Flajolet [12, Theorem 1]).

Lemma 2.4. [20, Lemma 3.2] For $p \neq 1,2, \ldots$, the function $\operatorname{Li}_{p}\left(e^{-z}\right)$ satisfies the singular expansion

$$
L i_{p}\left(e^{-z}\right) \sim \Gamma(1-p) z^{p-1}+\sum_{l=0}^{\infty}(-1)^{l} \varsigma(p-l) \frac{z^{l}}{l !}, \quad \text { as } z \rightarrow 0,
$$

where $\varsigma(z)$ denotes the Riemann zeta function. Then

Lemma 2.5. [20, Lemma 3.4] Let $|z| \leq \frac{\pi}{\sin \theta}$ with $\theta \in\left(\frac{\pi}{2}, \frac{5 \pi}{6}\right)$ and $-1<p<0$.

$$
L i_{p}\left(e^{-z}\right)=\Gamma(1-p) z^{p-1}+\sum_{l=0}^{\infty}(-1)^{l} \varsigma(p-l) \frac{z^{l}}{l !},
$$

converges absolutely.

Proof. [Proof of Lemma 2.3] We have, by the definition of the weights in (2.4), with $\zeta=e^{-z k}$,

$$
\begin{aligned}
\sum_{j=0}^{\infty} w_{j} \zeta^{j} & =\frac{1}{\Gamma(2-\alpha)}\left(\zeta^{-1}-2+\zeta\right)\left(\sum_{j=1}^{\infty} j^{1-\alpha} \zeta^{j}\right) \\
& =\frac{1}{\Gamma(2-\alpha)}\left(\left(e^{-z k}\right)^{-1}-2+e^{-z k}\right)\left(\sum_{j=1}^{\infty} j^{1-\alpha} \zeta^{j}\right) \\
& =\frac{1}{\Gamma(2-\alpha)}\left(\left(e^{-z k}\right)^{-1}-2+e^{-z k}\right) \operatorname{Li}_{\alpha-1}(\zeta),
\end{aligned}
$$

where $\operatorname{Li}_{\alpha-1}(\zeta)$ denotes the polylogarithm function. Thus, by Lemma 2.5,

$$
\operatorname{Li}_{\alpha-1}(\zeta)=\operatorname{Li}_{\alpha-1}\left(e^{-z k}\right)=\Gamma(2-\alpha)(z k)^{\alpha-2}+\sum_{l=0}^{\infty}(-1)^{l} \varsigma(1-\alpha-l) \frac{(z k)^{l}}{l !}
$$

where $\varsigma(z)$ denotes the Riemann zeta function. 
Hence, with some suitable constants $c_{2}, c_{3}, d_{0}, d_{1}, \ldots$,

$$
\begin{aligned}
\sum_{j=0}^{\infty} w_{j} \zeta^{j} & =\left((z k)^{2}+\frac{1}{12}(z k)^{4}+\ldots\right)\left((z k)^{\alpha-2}+d_{0}(z k)^{0}+d_{1}(z k)^{1}+\ldots\right) \\
& =(z k)^{\alpha}+c_{2}(z k)^{2}+c_{3}(z k)^{3}+\ldots
\end{aligned}
$$

Together these estimates complete the proof of Lemma 2.3.

Lemma 2.6. Let $\zeta=e^{-z k}$ and $z \in \Gamma_{k}$. Let $z_{k}$ be defined as in (2.5). Further we denote

$$
\mu(\zeta)=\frac{\zeta}{1-\zeta}\left(k z_{k}\right)
$$

and

$$
K(z)=z^{-1}\left(z^{\alpha}+A\right)^{-1} A .
$$

Then we have

$$
\begin{aligned}
& \mu\left(e^{-z k}\right)-1=O(z k), \quad \text { as } z k \rightarrow 0, \\
& c|z| \leq\left|z_{k}\right| \leq C|z|, \\
& \left\|K\left(z_{k}\right)-K(z)\right\| \leq C k^{2-\alpha}|z|^{-\alpha+1} . \\
& \left\|\mu(\zeta) K\left(z_{k}\right)-K(z)\right\| \leq C k|z|^{0} .
\end{aligned}
$$

Proof. We first show (2.9). It is sufficient to show

$$
\left|\mu\left(e^{-w}\right)-1\right| \leq C|w|, \quad \text { as } w \rightarrow 0 .
$$

Note that, by Lemma 2.3,

$$
\begin{aligned}
\mu\left(e^{-w}\right)-1 & =\frac{e^{-w}}{1-e^{-w}}\left(\sum_{j=0}^{\infty} w_{j}\left(e^{-w}\right)^{j}\right)^{\frac{1}{\alpha}}-1 \\
& =\frac{e^{-w}}{1-e^{-w}}\left(w^{\alpha}+c_{2} w^{2}+c_{3} w^{3}+\ldots\right)^{\frac{1}{\alpha}}-1 \\
& =e^{-w}\left(\frac{w}{1-e^{-w}}\right)\left(1+c_{2} w^{2-\alpha}+c_{3} w^{3-\alpha}+\ldots\right)^{\frac{1}{\alpha}}-1 \\
& =e^{-w}\left(\frac{w}{1-e^{-w}}\right)\left(1+c_{2} w^{2-\alpha}+\ldots\right)-1 .
\end{aligned}
$$

It is easy to see that $\lim _{w \rightarrow 0}\left(\mu\left(e^{-w}\right)-1\right)=0$, which implies that $\lim _{w \rightarrow 0} \frac{\mu\left(e^{-w}\right)-1}{w}$ exists. Hence (2.13) holds.

Next we show (2.10). Note that

$$
\frac{|z|}{\left|z_{k}\right|}=\frac{|z|}{\left|\frac{\delta\left(e^{-z k}\right)}{k}\right|}=\frac{|z k|}{\left|\delta\left(e^{-z k}\right)\right|} .
$$

To show (2.10), it suffices to prove $\frac{|z k|}{\left|\delta\left(e^{-z k}\right)\right|}$ has limit as $|z k| \rightarrow 0$, which follows from

$$
\begin{aligned}
\lim _{w \rightarrow 0} \frac{w}{\delta\left(e^{-w}\right)} & =\lim _{w \rightarrow 0} \frac{w}{\left(\sum_{j=0}^{\infty} w_{j}\left(e^{-w}\right)^{j}\right)^{\frac{1}{\alpha}}}=\lim _{w \rightarrow 0} \frac{w}{\left(w^{\alpha}+c_{2} w^{2}+\ldots\right)^{\frac{1}{\alpha}}} \\
& =\lim _{w \rightarrow 0} \frac{1}{\left(1+c_{2} w^{2-\alpha}+\ldots\right)^{\frac{1}{\alpha}}}=1 .
\end{aligned}
$$


Hence we have proved, for any fixed constant $M>0$, there exists a constant $C$ such that

$$
\frac{|z|}{\left|z_{k}\right|} \leq C, \forall|z k| \leq M
$$

Similarly we may show $\frac{\left|z_{k}\right|}{|z|} \leq C, \forall|z k| \leq M$. Thus we get (2.10).

We now show (2.11). Note that

$$
\begin{aligned}
z_{k}-z & =\frac{\delta\left(e^{-z k}\right)}{k}-z=\frac{\delta\left(e^{-z k}\right)-z k}{k}=\frac{\left(\sum_{j=0}^{\infty} w_{j}\left(e^{-z k}\right)^{j}\right)^{\frac{1}{\alpha}}-z k}{k} \\
& =\frac{\left((z k)^{\alpha}+c_{2} z^{2} k^{2}+\ldots\right)^{\frac{1}{\alpha}}-z k}{k}=\frac{(z k)\left(1+c_{2}(z k)^{2-\alpha}+\ldots\right)^{\frac{1}{\alpha}}-z k}{k} \\
& =\frac{(z k)\left(1+\frac{c_{2}}{\alpha}(z k)^{2-\alpha}+\ldots\right)-z k}{k}=O\left(k^{2-\alpha} z^{3-\alpha}\right), \quad \text { as } k z \rightarrow 0 .
\end{aligned}
$$

Thus we have, following the proof of $[30,(4.6)]$ and noting $\left\|K^{\prime}(z)\right\| \leq C|z|^{-2}$ in $[30,(3.12)]$,

$$
\left\|K\left(z_{k}\right)-K(z)\right\| \leq C|z|^{-2} k^{2-\alpha}|z|^{3-\alpha}=C k^{2-\alpha}|z|^{1-\alpha}
$$

Finally we show (2.12). Following the same proof as in the proof of [30, Lemma 4.3], we have

$$
\begin{gathered}
\left\|\mu(\zeta) K\left(z_{k}\right)-K(z)\right\| \leq\left\|(\mu(\zeta)-1) K\left(z_{k}\right)\right\|+\left\|K\left(z_{k}\right)-K(z)\right\| \\
\leq\left.(C \mid z k)|C| z\right|^{-1}+C k^{2-\alpha}|z|^{1-\alpha} \leq C k|z|^{0}+C k^{2-\alpha}|z|^{1-\alpha} \leq C k|z|^{0} .
\end{gathered}
$$

Together these estimates complete the proof of Lemma 2.6.

2.1.2. Proof of Theorem 1.1. In this subsection, we shall give the idea of the proof of Theorem 1.1. Then we follow the same idea to prove the error estimates for the modified L1 scheme in Theorem 1.3 later.

By using the Laplace transform and discrete Laplace transform, we have, see Jin et al. [20, Proof of Theorem 3.10],

$$
V\left(t_{n}\right)=-\frac{1}{2 \pi i} \int_{\Gamma} e^{t_{n} z} z^{-1}\left(z^{\alpha}+A\right)^{-1} A u_{0} d z
$$

and, with $z_{k}=\frac{\delta(\zeta)}{k}, \zeta=e^{-z k}$ defined by $(2.5)$,

$$
V^{n}=-\frac{1}{2 \pi i} \int_{\Gamma_{k}} e^{t_{n} z} \frac{\zeta}{1-\zeta}\left(k z_{k}\right) z_{k}^{-1}\left(z_{k}^{\alpha}+A\right)^{-1} A u_{0} d z
$$

where $\Gamma$ and $\Gamma_{k}$ are defined as in Lemma 2.1 .

Thus we have, subtracting (2.15) from (2.14),

$$
\begin{aligned}
V\left(t_{n}\right)-V^{n} & =\frac{1}{2 \pi i} \int_{\Gamma_{k}} e^{t_{n} z}\left(\mu(\zeta) K\left(z_{k}\right)-K(z)\right) u_{0} d z+\frac{1}{2 \pi i} \int_{\Gamma / \Gamma_{k}} e^{t_{n} z} K(z) u_{0} d z \\
& =I+I I
\end{aligned}
$$

where $\mu(\zeta)$ and $K(z)$ are defined by (2.7) and (2.8), respectively. 
For $I$, we have, by (2.12), with some suitable constant $c>0$,

$$
\begin{aligned}
\|I\| & \leq \frac{1}{2 \pi} \int_{\Gamma_{k}}\left|e^{t_{n} z}\right|\left\|\mu(\zeta) K\left(z_{k}\right)-K(z)\right\|\left\|u_{0}\right\||d z| \\
& \leq \frac{1}{2 \pi} \int_{\Gamma_{k}}\left|e^{t_{n} z}\right| C k\left\|u_{0}\right\||d z| \leq C k \int_{0}^{\infty} e^{-c t_{n} r} t_{n}^{-1} d\left(r t_{n}\right)\left\|u_{0}\right\| \leq C k t_{n}^{-1}\left\|u_{0}\right\| .
\end{aligned}
$$

For II, we have, by (1.4) and noting that $\left(z^{\alpha}+A\right)^{-1} A=I-z^{\alpha}\left(z^{\alpha}+A\right)^{-1}$, with some suitable constant $c>0$,

$$
\begin{aligned}
\|I I\| & \leq \frac{1}{2 \pi} \int_{\Gamma / \Gamma_{k}}\left|e^{t_{n} z}\right|\left\|u_{0}\right\|\left\|z^{-1}\left(z^{\alpha}+A\right)^{-1} A\right\|\left\|u_{0}\right\||d z|\left\|u_{0}\right\| \leq C \int_{\frac{1}{k}}^{\infty} e^{-c t_{n}|z|}|z|^{-1}|d z|\left\|u_{0}\right\| \\
& \leq C k \int_{\frac{1}{k}}^{\infty} e^{-c t_{n}|z|}|d z|\left\|u_{0}\right\| \leq C k t_{n}^{-1} \int_{0}^{\infty} e^{-c r} d r\left\|u_{0}\right\| \leq C k t_{n}^{-1}\left\|u_{0}\right\| .
\end{aligned}
$$

The proof of Theorem 1.1 is now complete.

REMARK 2.7. We remark that assuming that $u_{0} \in \mathcal{D}(A)$ rather than $u_{0} \in L_{2}(\Omega)$ reduces the singular behaviour of the error bound at $t=0$. We can prove the convergence order $O(k), 0<\alpha<1$ similarly, see Lubich et al. [30, p.16].

2.2. The modified $L 1$ scheme. In this section, we shall consider the modified L1 scheme (1.9)-(1.11) for solving (1.6) and prove that this scheme has the convergence rate $O\left(k^{2-\alpha}\right)$ for smooth and nonsmooth initial data.

The idea of introducing the correction term in the first step $n=1$ in (1.9) comes from Lubich et al. [30] where the authors introduced a modified scheme to construct second order time discretization scheme for solving an evolution equation with a positive-type memory term. To see this, let us write (1.6) into the equivalent form, with $0<\alpha<1$,

$$
V(t)+{ }_{0}^{R} D_{t}^{-\alpha}(A V(t))=-{ }_{0}^{R} D_{t}^{-\alpha}\left(A u_{0}\right), \quad \text { with } V(0)=0,
$$

where ${ }_{0}^{R} D_{t}^{-\alpha} V(t)$ denotes the Riemann-Liouville fractional integral. To obtain a higher order time discretization scheme for solving (2.16), following the idea in Lubich et al. [30], we may introduce the following modified time discretization scheme to approximate (2.16),

$$
V^{n}+q_{n}^{c}(A V)=-q_{n}^{c}\left(A u_{0}\right), \quad \text { with } V(0)=0,
$$

where $q_{n}^{c}(\varphi)$ is the modification of the quadrature formula approximating the RiemannLiouville fractional integral ${ }_{0}^{R} D_{t}^{-\alpha} \varphi$, defined by

$$
q_{n}^{c}(\varphi)=k^{-\alpha} \sum_{k=1}^{n} \beta_{n-j} \varphi^{j}+c_{0} \beta_{n-1} \varphi^{0}, \quad \text { with } c_{0}=1 / 2 .
$$

Here $\beta_{0}, \beta_{1}, \ldots$ are generated by some function $\hat{\beta}(\zeta)=\sum_{j=0}^{\infty} \beta_{j} \zeta^{j}$.

We have the following lemma.

Lemma 2.8. Assume that $\hat{\beta}(\zeta)=\left(\sum_{j=0}^{\infty} w_{j} \zeta^{j}\right)^{-1}$, where $w_{j}, j=0,1,2, \ldots$ are defined in (2.4). Then the modified L1 scheme (1.9)-(1.11) is equivalent to (2.17).

Proof. Denote

$$
a_{n}= \begin{cases}1+c_{0}, \quad c_{0}=1 / 2, & \text { for } n=1, \\ 1, & \text { for } n \geq 2 .\end{cases}
$$


The time discretization problem of (1.9)-(1.11) can then be written as

$$
k^{-\alpha} \sum_{j=1}^{n} w_{n-j} V^{j}+A V^{n}=\left(-A u_{0}\right) a_{n} .
$$

Taking the discrete Laplace transform in both sides, we have

$$
\sum_{n=1}^{\infty}\left(k^{-\alpha} \sum_{j=1}^{n} w_{n-j} V^{j}\right) \zeta^{n}+\sum_{n=1}^{\infty}\left(A V^{n}\right) \zeta^{n}=\left(-A u_{0}\right) \sum_{n=1}^{\infty}\left(a_{n} \zeta^{n}\right)
$$

Note that

$$
\sum_{n=1}^{\infty}\left(\sum_{j=1}^{n} w_{n-j} V^{j}\right) \zeta^{n}=\left(\sum_{j=0}^{\infty} w_{j} \zeta^{j}\right)\left(V^{1} \zeta^{1}+V^{2} \zeta^{2}+\ldots\right),
$$

we have, with $\hat{w}(\zeta)=\sum_{j=0}^{\infty} w_{j} \zeta^{j}, \hat{V}(\zeta)=\sum_{j=0}^{\infty} V^{j} \zeta^{j}$,

$$
k^{-\alpha} \hat{w}(\zeta) \hat{V}(\zeta)+A \hat{V}(\zeta)=\left(-A u_{0}\right)\left(\frac{\zeta}{1-\zeta}+c_{0} \zeta\right) .
$$

By the assumption for $\hat{\beta}(\zeta)$, we have

$$
\hat{V}(\zeta)+k^{\alpha} \hat{\beta}(\zeta) A \hat{V}(\zeta)=k^{\alpha} \hat{\beta}(\zeta)\left(-A u_{0}\right)\left(\frac{\zeta}{1-\zeta}+c_{0} \zeta\right)
$$

Thus we get

$$
\begin{aligned}
& \sum_{n=1}^{\infty} V^{n} \zeta^{n}+k^{\alpha} \sum_{n=1}^{\infty}\left(\sum_{j=1}^{n} \beta_{n-j} A V^{j}\right) \zeta^{n} \\
= & -k^{\alpha} \sum_{n=1}^{\infty}\left(\sum_{j=1}^{n} \beta_{n-j} A u_{0}\right) \zeta^{n}-k^{\alpha} \sum_{n=1}^{\infty}\left(c_{0} \beta_{n-1} A u_{0}\right) \zeta^{n} .
\end{aligned}
$$

Hence

$$
V^{n}+k^{\alpha} \sum_{j=1}^{n} \beta_{n-j} A V^{j}=-k^{\alpha} \sum_{j=1}^{n} \beta_{n-j} A u_{0}-k^{\alpha} c_{0} \beta_{n-1} A u_{0}, n \geq 1,
$$

which is $(2.17)$.

Together these estimates complete the proof of Lemma 2.8.

REMARK 2.9. From Lemma 2.8, we note that the correction on the first step $n=1$ in (1.9)-(1.11) is equivalent to the correction in (2.17). Therefore we see that the modified L1 scheme (1.9)-(1.11) is actually equivalent to the modified scheme (2.17) which has been used to improve the convergence rate of the time discretization scheme for solving an evolution equation with a positive-type memory term in Lubich et al. [30].

2.2.1. Proof of Theorem 1.3. In this subsection, we shall prove Theorem 1.3 for the error estimates of the modified L1 scheme (1.9)-(1.11). To prove Theorem 1.3, we need the following lemma. 
Lemma 2.10. Let $\zeta=e^{-z k}$ and $z \in \Gamma_{k}$. Let $z_{k}$ and $K(z)$ be defined as in (2.5), (2.8), respectively. Further we denote, with $c_{0}=1 / 2$,

$$
\bar{\mu}(\zeta)=\left(\frac{\zeta}{1-\zeta}+c_{0} \zeta\right) \delta(\zeta)
$$

where $\delta(\zeta)$ is defined in (2.5). Then we have

$$
\begin{aligned}
& \bar{\mu}\left(e^{-z k}\right)-1=O\left((z k)^{2-\alpha}\right), \quad \text { as } z k \rightarrow 0, \\
& \left\|\bar{\mu}(\zeta) K\left(z_{k}\right)-K(z)\right\| \leq C k^{2-\alpha}|z|^{1-\alpha} .
\end{aligned}
$$

Proof. We first show (2.22). It is sufficient to show

$$
\left|\bar{\mu}\left(e^{-w}\right)-1\right| \leq C|w|^{2-\alpha}, \quad \text { as } w \rightarrow 0 .
$$

Note that, by Lemma 2.3,

$$
\begin{aligned}
\bar{\mu}\left(e^{-w}\right)-1 & =\left(\frac{e^{-w}}{1-e^{-w}}+c_{0} e^{-w}\right)\left(\sum_{j=0}^{\infty} w_{j}\left(e^{-w}\right)^{j}\right)^{\frac{1}{\alpha}}-1 \\
& =\left(\frac{e^{-w}}{1-e^{-w}}+c_{0} e^{-w}\right)\left(w^{\alpha}+c_{2} w^{2}+c_{3} w^{3}+\ldots\right)^{\frac{1}{\alpha}}-1 \\
& =\left(e^{-w}+c_{0} e^{-w}\left(1-e^{-w}\right)\right)\left(\frac{w}{1-e^{-w}}\right)\left(1+c_{2} w^{2-\alpha}+c_{3} w^{3-\alpha}+\ldots\right)^{\frac{1}{\alpha}}-1 \\
& =\left(e^{-w}+c_{0} e^{-w}\left(1-e^{-w}\right)\right)\left(\frac{w}{1-e^{-w}}\right)\left(1+c_{2} w^{2-\alpha}+\ldots\right)-1 \\
& =f_{1}(w) f_{2}(w) f_{3}(w)-1,
\end{aligned}
$$

where $f_{1}(w)=e^{-w}+c_{0} e^{-w}\left(1-e^{-w}\right), \quad f_{2}(w)=\frac{w}{1-e^{-w}}$, and $f_{3}(w)=1+c_{2} w^{2-\alpha}+\ldots$ Here $c_{2}, c_{3}, \ldots$ denote generic constants, which may differ at different occurrences.

We now have

$$
\begin{aligned}
\lim _{w \rightarrow 0} \frac{\bar{\mu}\left(e^{-w}\right)-1}{w^{2-\alpha}} & =\lim _{w \rightarrow 0} \frac{F(w)+f_{1}(w)\left(f_{2}(w) f_{3}^{\prime}(w)\right)}{(2-\alpha) w^{1-\alpha}} \\
& =\lim _{w \rightarrow 0} \frac{F(w)+f_{1}(w) f_{2}(w)\left(c_{2} w^{1-\alpha}+\ldots\right)}{(2-\alpha) w^{1-\alpha}} .
\end{aligned}
$$

Here

$$
\begin{aligned}
F(w) & =f_{1}^{\prime}(w) f_{2}(w) f_{3}(w)+f_{1}(w) f_{2}^{\prime}(w) f_{3}(w) \\
& =\left(e^{-w}(-1)+c_{0} e^{-w}(-1)\left(1-e^{-w}\right)+c_{0} e^{-w} e^{-w}\right) f_{2}(w) f_{3}(w) \\
& +\left(e^{-w}+c_{0} e^{-w}\left(1-e^{-w}\right)\right)\left(\frac{\left(1-e^{-w}\right)-w e^{-w}}{\left(1-e^{-w}\right)^{2}}\right) f_{3}(w)
\end{aligned}
$$

With $c_{0}=1 / 2$, it is easy to see that $\lim _{w \rightarrow 0} F(w)=O(w)$. Further we have $\lim _{w \rightarrow 0} f_{1}(w) f_{2}(w)=C$. Thus the following limit exists

$$
\lim _{w \rightarrow 0} \frac{\bar{\mu}\left(e^{-w}\right)-1}{w^{2-\alpha}}=\lim _{w \rightarrow 0} \frac{F(w)+f_{1}(w) f_{2}(w)\left(c_{2} w^{1-\alpha}+\ldots\right)}{(2-\alpha) w^{1-\alpha}},
$$


which shows (2.24).

Finally we show (2.23). Following the same proof as in the proof of [30, Lemma 4.3], we have

$$
\begin{aligned}
\left\|\bar{\mu}(\zeta) K\left(z_{k}\right)-K(z)\right\| & \leq\left\|(\bar{\mu}(\zeta)-1) K\left(z_{k}\right)\right\|+\left\|K\left(z_{k}\right)-K(z)\right\| \\
& \leq|z k|^{2-\alpha} C|z|^{-1}+C k^{2-\alpha}|z|^{1-\alpha} \leq C k^{2-\alpha}|z|^{1-\alpha} .
\end{aligned}
$$

Together these estimates complete the proof of Lemma 2.10.

口

Proof. [Proof of Theorem 1.3] Following the same argument as in the proof of Theorem 1.1, we may obtain this time

$$
\begin{aligned}
V\left(t_{n}\right)-V^{n}= & \frac{1}{2 \pi i} \int_{\Gamma_{k}} e^{t_{n} z}\left(\bar{\mu}(\zeta) K\left(z_{k}\right)-K(z)\right) u_{0} d z \\
& +\frac{1}{2 \pi i} \int_{\Gamma / \Gamma_{k}} e^{t_{n} z} K(z) u_{0} d z=I+I I,
\end{aligned}
$$

where $K(z)$ and $\bar{\mu}(\zeta)$ are defined by (2.8) and (2.21), respectively. Then we have, by (2.23), with some suitable constant $c>0$,

$$
\begin{aligned}
\|I\| & \leq \frac{1}{2 \pi} \int_{\Gamma_{k}}\left|e^{t_{n} z}\right|\left\|\bar{\mu}(\zeta) K\left(z_{k}\right)-K(z)\right\|\left\|u_{0}\right\||d z| \\
& \leq \frac{1}{2 \pi} \int_{\Gamma_{k}}\left|e^{t_{n} z}\right| C\left(k^{2-\alpha}|z|^{1-\alpha}\right)\left\|u_{0}\right\||d z| \\
& \leq C k^{2-\alpha} \int_{0}^{\infty} e^{-c t_{n} r}\left(t_{n} r\right)^{1-\alpha} d\left(r t_{n}\right) t_{n}^{\alpha-1} t_{n}^{-1}\left\|u_{0}\right\| \\
& \leq C k^{2-\alpha} t_{n}^{\alpha-2}\left\|u_{0}\right\| \leq C k^{2-\alpha} t_{n}^{\alpha-2}\left\|u_{0}\right\| .
\end{aligned}
$$

For II, we have, by (1.4) and noting that $\left(z^{\alpha}+A\right)^{-1} A=I-z^{\alpha}\left(z^{\alpha}+A\right)^{-1}$, with some suitable constant $c>0$,

$$
\begin{aligned}
\|I I\| & \leq \frac{1}{2 \pi} \int_{\Gamma / \Gamma_{k}}\left|e^{t_{n} z}\right|\left\|u_{0}\right\|\left\|z^{-1}\left(z^{\alpha}+A\right)^{-1} A\right\|\left\|u_{0}\right\||d z|\left\|u_{0}\right\| \leq C \int_{\frac{1}{k}}^{\infty} e^{-c t_{n}|z|}|z|^{-1}|d z|\left\|u_{0}\right\| \\
& \leq C \int_{\frac{1}{k}}^{\infty} e^{-c t_{n}|z|}|z|^{-(2-\alpha)}|z|^{-\alpha+1}|d z|\left\|u_{0}\right\| \leq C k^{2-\alpha} \int_{\frac{1}{k}}^{\infty} e^{-c t_{n}|z|}|z|^{1-\alpha}|d z|\left\|u_{0}\right\| \\
& \leq C k^{2-\alpha} t_{n}^{\alpha-2} \int_{0}^{\infty} e^{-c r} r^{-\alpha+1} d r\left\|u_{0}\right\| \leq C k^{2-\alpha} t_{n}^{\alpha-2}\left\|u_{0}\right\| .
\end{aligned}
$$

The proof of Theorem 1.3 is now complete.

REMARK 2.11. We remark that assuming that $u_{0} \in \mathcal{D}(A)$ rather than $u_{0} \in$ $L_{2}(\Omega)$ reduces the singular behavior of the error bound at $t=0$. We can prove the convergence order $O\left(k^{2-\alpha}\right), 0<\alpha<1$ similarly, see Lubich et al. [30, p.16]

3. The inhomogeneous problem. In this section we will consider the error estimates of the time stepping method (1.12)-(1.13) for solving the inhomogeneous problem (1.5) and prove Theorem 1.4. To do this, we need the following lemma.

Lemma 3.1. Let $z_{k}$ be defined as in (2.5). We have, with $\zeta=e^{-z k}$,

$$
\left\|\left(z^{\alpha}+A\right)^{-1} z^{-2}-\left(z_{k}^{\alpha}+A\right)^{-1}\left(k \sum_{n=1}^{\infty} t_{n} \zeta^{n}\right)\right\| \leq C k^{2-\alpha}|z|^{-2 \alpha} .
$$


Proof. We have

$$
\begin{aligned}
& \left\|\left(z^{\alpha}+A\right)^{-1} z^{-2}-\left(z_{k}^{\alpha}+A\right)^{-1}\left(k \sum_{n=1}^{\infty} t_{n} \zeta^{n}\right)\right\| \\
& \leq\left\|\left(z^{\alpha}+A\right)^{-1} z^{-2}-\left(z_{k}^{\alpha}+A\right)^{-1} z_{k}^{-2}\right\|+\left\|\left(z_{k}^{\alpha}+A\right)^{-1} z_{k}^{-2}\left(1-z_{k}^{2} k \sum_{n=1}^{\infty} t_{n} \zeta^{n}\right)\right\| .
\end{aligned}
$$

It is easy to show that

$$
\left\|1-z_{k}^{2} k \sum_{n=1}^{\infty} t_{n} \zeta^{n}\right\| \leq C|z k|^{2-\alpha} .
$$

The rest of the proof of Lemma 3.1 follows from the arguments for the proofs of (2.11) and (2.12).

口

Proof. [Proof of Theorem 1.4] The proof is following the arguments developed in [21] and [22] for the time fractional diffusion problem in the inhomogeneous case.

Denote

$$
f(t)=f(0)+R(t), \quad R(t)=t f^{\prime}(0)+\left(t * f^{\prime \prime}\right)(t) .
$$

Here $f * g$ denotes the convolution of $f$ and $g$.

Taking the Laplace transform in (1.5), we have

$$
z^{\alpha} \hat{V}(z)+A \hat{V}(z)=-A u_{0} z^{-1}+\hat{f}(z)=-A u_{0} z^{-1}+f(0) z^{-1}+\hat{R}(z),
$$

which implies that

$$
V(t)=\frac{1}{2 \pi i} \int_{\Gamma} e^{z t}\left(\left(z^{\alpha}+A\right)^{-1} z^{-1}\left(-A u_{0}+f(0)\right)+\left(z^{\alpha}+A\right)^{-1} \hat{R}(z)\right) d z .
$$

Taking the discrete Laplace transform in (1.12)-(1.13), we have

$$
\begin{aligned}
& \sum_{n=1}^{\infty}\left(k^{-\alpha} \sum_{j=1}^{n} w_{n-j} V^{j}\right) \zeta^{n}+\sum_{n=1}^{\infty}\left(A V^{n}\right) \zeta^{n} \\
& =\sum_{n=1}^{\infty}\left(-A u_{0}+f(0)\right) \zeta^{n}+\sum_{n=1}^{\infty} R\left(t_{n}\right) \zeta^{n}+c_{0}\left(-A u_{0}+f(0)\right) \zeta
\end{aligned}
$$

which implies that

$$
\begin{aligned}
V^{n} & =\frac{1}{2 \pi i} \int_{\Gamma_{k}} e^{z t_{n}}\left(z_{k}^{\alpha}+A\right)^{-1} z_{k}^{-1} \bar{\mu}\left(e^{-z k}\right)\left(-A u_{0}+f(0)\right) d z \\
& +\frac{1}{2 \pi i} \int_{\Gamma_{k}} e^{z t_{n}}\left(z_{k}^{\alpha}+A\right)^{-1} k\left(\sum_{n=1}^{\infty} R\left(t_{n}\right) \zeta^{n}\right) d z
\end{aligned}
$$

where $\bar{\mu}(\zeta)$ and $z_{k}$ are defined by (2.21) and (2.5), respectively. Thus we have

$$
V\left(t_{n}\right)-V^{n}=I_{1}+I_{2}
$$


where

$$
\begin{aligned}
I_{1}= & \frac{1}{2 \pi i} \int_{\Gamma / \Gamma_{k}} e^{z t_{n}}\left(z^{\alpha}+A\right)^{-1} z^{-1}\left(-A u_{0}+f(0)\right) d z \\
& +\frac{1}{2 \pi i} \int_{\Gamma_{k}} e^{z t_{n}}\left(\left(z^{\alpha}+A\right)^{-1} z^{-1}-\left(z_{k}^{\alpha}+A\right)^{-1} z_{k}^{-1} \bar{\mu}\left(e^{-z k}\right)\right)\left(-A u_{0}+f(0)\right) d z, \\
I_{2}= & \frac{1}{2 \pi i} \int_{\Gamma} e^{z t_{n}}\left(\left(z^{\alpha}+A\right)^{-1} z^{-1}\right)(z \hat{R}(z)) d z \\
& -\frac{1}{2 \pi i} \int_{\Gamma_{k}} e^{z t_{n}}\left(\left(z_{k}^{\alpha}+A\right)^{-1} z_{k}^{-1}\left(z_{k} k \sum_{n=1}^{\infty} R\left(t_{n}\right) \zeta^{n}\right)\right) d z .
\end{aligned}
$$

For $I_{1}$, we have, following the argument in the proof of Theorem 1.3,

$$
\left\|I_{1}\right\| \leq C k^{2-\alpha} t_{n}^{\alpha-2}\left\|u_{0}\right\|+C k^{2-\alpha} t_{n}^{2 \alpha-2}\|f(0)\| .
$$

For $I_{2}$, noting that $R(t)=R^{1}(t)+R^{2}(t)$, where $R^{1}(t)=t f^{\prime}(0)$ and $R^{2}(t)=$ $\left(t * f^{\prime \prime}\right)(t)$, we may write $I_{2}$ as

$$
I_{2}=I_{2}^{1}+I_{2}^{2}
$$

where

$$
\begin{aligned}
I_{2}^{1}= & \frac{1}{2 \pi i} \int_{\Gamma} e^{z t_{n}}\left(\left(z^{\alpha}+A\right)^{-1} z^{-1}\right)\left(z \hat{R}^{1}(z)\right) d z \\
& -\frac{1}{2 \pi i} \int_{\Gamma_{k}} e^{z t_{n}}\left(\left(z_{k}^{\alpha}+A\right)^{-1} z_{k}^{-1}\left(z_{k} k \sum_{n=1}^{\infty} R^{1}\left(t_{n}\right) \zeta^{n}\right)\right) d z \\
I_{2}^{2}= & \frac{1}{2 \pi i} \int_{\Gamma} e^{z t_{n}}\left(\left(z^{\alpha}+A\right)^{-1} z^{-1}\right)\left(z \hat{R}^{2}(z)\right) d z \\
& -\frac{1}{2 \pi i} \int_{\Gamma_{k}} e^{z t_{n}}\left(\left(z_{k}^{\alpha}+A\right)^{-1} z_{k}^{-1}\left(z_{k} k \sum_{n=1}^{\infty} R^{2}\left(t_{n}\right) \zeta^{n}\right)\right) d z .
\end{aligned}
$$

For $I_{2}^{1}$, we have

$$
\begin{aligned}
\left\|I_{2}^{1}\right\|= & \| \frac{1}{2 \pi i} \int_{\Gamma} e^{z t_{n}}\left(\left(z^{\alpha}+A\right)^{-1} z^{-2}\right) d z f^{\prime}(0) \\
& -\frac{1}{2 \pi i} \int_{\Gamma_{k}} e^{z t_{n}}\left(\left(z_{k}^{\alpha}+A\right)^{-1}\left(k \sum_{n=1}^{\infty} t_{n} \zeta^{n}\right)\right) d z f^{\prime}(0) \| \\
= & \| \frac{1}{2 \pi i} \int_{\Gamma / \Gamma_{k}} e^{z t_{n}}\left(\left(z^{\alpha}+A\right)^{-1} z^{-2}\right) d z f^{\prime}(0) \\
& -\frac{1}{2 \pi i} \int_{\Gamma_{k}} e^{z t_{n}}\left(\left(z^{\alpha}+A\right)^{-1} z^{-2}-\left(z_{k}^{\alpha}+A\right)^{-1}\left(k \sum_{n=1}^{\infty} t_{n} \zeta^{n}\right)\right) d z f^{\prime}(0) \| .
\end{aligned}
$$

By Lemma 3.1, we have

$$
\left\|I_{2}^{1}\right\| \leq C k^{2-\alpha} t_{n}^{2 \alpha-1}\left\|f^{\prime}(0)\right\| .
$$

For $I_{2}^{2}$, we have, following the arguments as in Jin et al. [21], [22],

$$
\left\|I_{2}^{2}\right\| \leq C k^{2-\alpha} \int_{0}^{t_{n}}\left(t_{n}-s\right)^{2 \alpha-1}\left\|f^{\prime \prime}(s)\right\| d s .
$$


Together these estimates complete the proof of Theorem 1.4.

REMARK 3.2. We remark that assuming that $u_{0} \in \mathcal{D}(A)$ rather than $u_{0} \in L_{2}(\Omega)$ reduces the singular behavior of the error bound at $t=0$. Let $V\left(t_{n}\right)$ and $V^{n}$ be the solutions of (1.5) and (1.12)-(1.13), respectively. Let $u_{0} \in \mathcal{D}(A)$. Then we can prove, following the argument of the proof in Jin et al. [22, Theorem 2.2], with $0<\alpha<1$,

$$
\left\|V\left(t_{n}\right)-V^{n}\right\| \leq C k^{2-\alpha}\left(t_{n}^{2 \alpha-2}\left\|f(0)+A u_{0}\right\|+t_{n}^{2 \alpha-1}\left\|f^{\prime}(0)\right\|+\int_{0}^{t_{n}}\left(t_{n}-s\right)^{2 \alpha-1}\left\|f^{\prime \prime}(s)\right\| d s\right) .
$$

Thus we observe that if $f(0)+A u_{0}=0$ and $f^{\prime}(0)=0$, we obtain the uniform convergence rate $O\left(k^{2-\alpha}\right)$.

4. Numerical simulations. In this section, we will consider the experimentally determined convergence rates of the L1 and the modified L1 schemes for smooth and nonsmooth data in both homogeneous and inhomogeneous cases.

EXAMPLE 4.1. Let us consider the following homogeneous problem

$$
\begin{aligned}
& { }_{0}^{C} D_{t}^{\alpha} u(x, t)-u_{x x}=0, \quad 0<x<1, \quad t>0, \\
& u(0, t)=u(1, t)=0, \\
& u(x, 0)=u_{0}(x)
\end{aligned}
$$

where $u_{0}(x)=x(1-x)$ or $u_{0}(x)=\chi_{(0,1 / 2)}$.

Let $0<t_{0}<t_{1}<\ldots t_{N}=T$ be the time partition on $[0, T]$ and $k$ the time step size. Let $N_{h}$ be a positive integer. Let $0=x_{0}<x_{1}<x_{2}<\ldots x_{N_{h}}=1$ be the space partition on $[0,1]$ and $h$ the space step size. We will use the linear finite element method to consider the spatial discretization.

We first consider the scheme (1.7) and the convergence rate was proved to be $O(k)$ for both smooth and nonsmooth data in [20]. To observe this convergence order, we first calculate the reference solution $u_{\text {ref }}(t)$ at $T=1$ with $h_{\text {ref }}=2^{-6}$ and $k_{r e f}=2^{-10}$. We then use $h=2^{-6}$ and $k=\kappa * k_{r e f}$ with $\kappa=\left[2^{2}, 2^{3}, 2^{4}, 2^{5}, 2^{6}\right]$ to obtain the approximate solutions $u(t)$ at $T=1$. We choose the smooth and nonsmooth initial data (a) $u_{0}=x(1-x)$ and (b) $u_{0}=\chi_{(0,1 / 2)}$. We obtain the following results which are consistent with the Table $1 \mathrm{in}$ [20]. The convergence rate indeed is almost $O(k)$ for the different $\alpha \in(0,1)$ for smooth and nonsmooth initial data.

\begin{tabular}{|c|c|ccccc|c|}
\hline$\alpha$ & & $k=2^{-8}$ & $k=2^{-7}$ & $k=2^{-6}$ & $k=2^{-5}$ & $k=2^{-4}$ & Rate \\
\hline 0.1 & $(a)$ & $0.0212 \mathrm{e}-4$ & $0.0496 \mathrm{e}-4$ & $0.1067 \mathrm{e}-4$ & $0.2218 \mathrm{e}-4$ & $0.4564 \mathrm{e}-4$ & 1.1063 \\
& $(b)$ & $0.0055 \mathrm{e}-3$ & $0.0127 \mathrm{e}-3$ & $0.0274 \mathrm{e}-3$ & $0.0570 \mathrm{e}-3$ & $0.1172 \mathrm{e}-3$ & 1.1063 \\
\hline 0.3 & $(a)$ & $0.0056 \mathrm{e}-3$ & $0.0130 \mathrm{e}-3$ & $0.0280 \mathrm{e}-3$ & $0.0585 \mathrm{e}-3$ & $0.1209 \mathrm{e}-3$ & 1.1100 \\
& $(b)$ & $0.0143 \mathrm{e}-3$ & $0.0333 \mathrm{e}-3$ & $0.0718 \mathrm{e}-3$ & $0.1479 \mathrm{e}-3$ & $0.3094 \mathrm{e}-3$ & 1.1099 \\
\hline 0.8 & $(a)$ & $0.0078 \mathrm{e}-3$ & $0.0185 \mathrm{e}-3$ & $0.0403 \mathrm{e}-3$ & $0.0857 \mathrm{e}-3$ & $0.1824 \mathrm{e}-3$ & 1.1359 \\
& $(b)$ & $0.0198 \mathrm{e}-3$ & $0.0466 \mathrm{e}-3$ & $0.1017 \mathrm{e}-3$ & $0.2160 \mathrm{e}-3$ & $0.4595 \mathrm{e}-3$ & 1.1350 \\
\hline 0.9 & $(a)$ & $0.0054 \mathrm{e}-3$ & $0.0128 \mathrm{e}-3$ & $0.0284 \mathrm{e}-3$ & $0.0621 \mathrm{e}-3$ & $0.1404 \mathrm{e}-3$ & 1.1766 \\
& $(b)$ & $0.0134 \mathrm{e}-3$ & $0.0320 \mathrm{e}-3$ & $0.0708 \mathrm{e}-3$ & $0.1546 \mathrm{e}-3$ & $0.3490 \mathrm{e}-3$ & 1.1757 \\
\hline
\end{tabular}

TABLE 1

Time convergence rates with the different $\alpha$ for the L1 scheme (1.7) in Example 4.1 
We next consider the modified L1 scheme (1.9)-(1.11). By Theorem 1.3, the convergence rate of the modified L1 scheme (1.9)-(1.11) is $O\left(k^{2-\alpha}\right)$ for smooth and nonsmooth initial data. We use the same notations as in Table 1 and we obtain the following results in Table 2.

We found that the modified L1 scheme has the better accuracy than the L1 scheme and the errors are about $1 e-05$ or $1 e-04$ for all $\alpha \in(0,1)$. The errors of the L1 scheme are only $1 e-03$. For the convergence rates, when $\alpha<1 / 2$, we observe that, in Table 2, the convergence rates are almost 2 which is better than the theoretical results $2-\alpha$. However when $\alpha>1 / 2$, the convergence rates are almost $2-\alpha$ as we expected.

\begin{tabular}{|c|c|ccccc|c|}
\hline$\alpha$ & & $k=2^{-8}$ & $k=2^{-7}$ & $k=2^{-6}$ & $k=2^{-5}$ & $k=2^{-4}$ & Rate \\
\hline 0.1 & $(a)$ & $0.0013 \mathrm{e}-5$ & $0.0055 \mathrm{e}-5$ & $0.0233 \mathrm{e}-5$ & $0.0985 \mathrm{e}-5$ & $0.4265 \mathrm{e}-5$ & 2.0985 \\
& $(b)$ & $0.0018 \mathrm{e}-5$ & $0.0078 \mathrm{e}-5$ & $0.0322 \mathrm{e}-5$ & $0.1333 \mathrm{e}-5$ & $0.5658 \mathrm{e}-5$ & 2.0668 \\
\hline 0.3 & $(a)$ & $0.0013 \mathrm{e}-5$ & $0.0064 \mathrm{e}-5$ & $0.0291 \mathrm{e}-5$ & $0.1302 \mathrm{e}-5$ & $0.5891 \mathrm{e}-5$ & 2.1914 \\
& $(b)$ & $0.0004 \mathrm{e}-4$ & $0.0017 \mathrm{e}-4$ & $0.0076 \mathrm{e}-4$ & $0.0339 \mathrm{e}-4$ & $0.1527 \mathrm{e}-4$ & 2.1839 \\
\hline 0.8 & $(a)$ & $0.0079 \mathrm{e}-4$ & $0.0201 \mathrm{e}-4$ & $0.0462 \mathrm{e}-4$ & $0.0981 \mathrm{e}-4$ & $0.1782 \mathrm{e}-4$ & 1.1223 \\
& $(b)$ & $0.0196 \mathrm{e}-4$ & $0.0496 \mathrm{e}-4$ & $0.1140 \mathrm{e}-4$ & $0.2421 \mathrm{e}-4$ & $0.4407 \mathrm{e}-4$ & 1.1230 \\
\hline 0.9 & $(a)$ & $0.0141 \mathrm{e}-4$ & $0.0345 \mathrm{e}-4$ & $0.0778 \mathrm{e}-4$ & $0.1687 \mathrm{e}-4$ & $0.3484 \mathrm{e}-4$ & 1.1573 \\
& $(b)$ & $0.0347 \mathrm{e}-4$ & $0.0851 \mathrm{e}-4$ & $0.1920 \mathrm{e}-4$ & $0.4162 \mathrm{e}-4$ & $0.8597 \mathrm{e}-4$ & 1.1572 \\
\hline
\end{tabular}

TABLE 2 4.1

Time convergence rates with the different $\alpha$ for the modified L1 scheme (1.9)-(1.11) in Example

EXAMPLE 4.2. Let us consider the following inhomogeneous problem

$$
\begin{aligned}
& { }_{0}^{C} D_{t}^{\alpha} u(x, t)-u_{x x}=f(x, t), \quad 0<x<1, \quad t>0, \\
& u(0, t)=u(1, t)=0, \\
& u(x, 0)=x(1-x),
\end{aligned}
$$

where $f(x, t)=\sin (t)\left(1+\chi_{(0,1 / 2)}(x)\right)$. Here the source term $f$ is smooth in time, therefore Theorem 1.4 is applicable.

We use the same notations as in Example 4.1. We first consider the L1 scheme (1.12)-(1.13) (i.e., $c_{0}=0$ ) and we find that the experimentally determined convergence rate is almost $O(k)$ for the different values of $\alpha \in(0,1)$, see Table 3 .

\begin{tabular}{|c|ccccc|c|}
\hline$\alpha$ & $k=2^{-8}$ & $k=2^{-7}$ & $k=2^{-6}$ & $k=2^{-5}$ & $k=2^{-4}$ & Rate \\
\hline 0.1 & $0.0212 \mathrm{e}-4$ & $0.0492 \mathrm{e}-4$ & $0.1050 \mathrm{e}-4$ & $0.2161 \mathrm{e}-4$ & $0.4370 \mathrm{e}-4$ & 1.10929 \\
\hline 0.3 & $0.0055 \mathrm{e}-3$ & $0.0127 \mathrm{e}-3$ & $0.0270 \mathrm{e}-3$ & $0.0553 \mathrm{e}-3$ & $0.1111 \mathrm{e}-3$ & 1.0859 \\
\hline 0.8 & $0.0353 \mathrm{e}-3$ & $0.0761 \mathrm{e}-3$ & $0.1486 \mathrm{e}-4$ & $0.2811 \mathrm{e}-3$ & $0.5570 \mathrm{e}-3$ & 0.9953 \\
\hline 0.9 & $0.0169 \mathrm{e}-4$ & $0.0452 \mathrm{e}-4$ & $0.1083 \mathrm{e}-4$ & $0.2350 \mathrm{e}-4$ & $0.4200 \mathrm{e}-4$ & 1.1589 \\
\hline
\end{tabular}


We then consider the modified L1 scheme (1.12)-(1.13) (i.e., $\left.c_{0}=1 / 2\right)$. By Theorem 1.4, the convergence rate of the modified L1 scheme (1.9)-(1.11) is $O\left(k^{2-\alpha}\right)$ for the sufficiently smooth source term $f$. This is fully supported by the numerical results in Table 4.

\begin{tabular}{|c|ccccc|c|}
\hline$\alpha$ & $k=2^{-8}$ & $k=2^{-7}$ & $k=2^{-6}$ & $k=2^{-5}$ & $k=2^{-4}$ & Rate \\
\hline 0.1 & $0.0020 \mathrm{e}-5$ & $0.0078 \mathrm{e}-5$ & $0.0293 \mathrm{e}-5$ & $0.1094 \mathrm{e}-5$ & $0.4139 \mathrm{e}-5$ & 1.9239 \\
\hline 0.3 & $0.0011 \mathrm{e}-4$ & $0.0038 \mathrm{e}-4$ & $0.0131 \mathrm{e}-4$ & $0.0448 \mathrm{e}-4$ & $0.1562 \mathrm{e}-4$ & 1.7972 \\
\hline 0.8 & $0.0273 \mathrm{e}-4$ & $0.0713 \mathrm{e}-4$ & $0.1732 \mathrm{e}-4$ & $0.4109 \mathrm{e}-4$ & $0.9776 \mathrm{e}-4$ & 1.2903 \\
\hline 0.9 & $0.0057 \mathrm{e}-3$ & $0.0139 \mathrm{e}-3$ & $0.0315 \mathrm{e}-3$ & $0.0687 \mathrm{e}-3$ & $0.1474 \mathrm{e}-3$ & 1.1761 \\
\hline
\end{tabular}

TABLE 4

Time convergence rates for the L1 scheme (1.12)-(1.13) (i.e., $\left.c_{0}=1 / 2\right)$ in Example 4.2

\section{REFERENCES}

[1] E. E. Adams and L. W. Gelhar, Field study of dispersion in a heterogeneous aquifer: 2. Spatial moments analysis, Water Res. Research, 28(1992), pp. 3293-3307.

[2] E. Bazhlekova, B. Jin, R. Lazarov, and Z. Zhou, An analysis of the Rayleigh-Stokes problem for a generalized second-grade fluid, Numer. Math., 131(2015), pp. 1-31.

[3] W. Cao, F. Zeng, Z. Zhang, and G. E. Karniadakis, Implicit-explicit difference schemes for nonlinear fractional differential equations with nonsmooth solutions, SIAM J. Sci. Comput., 38(2016), pp. A3070-A3093.

[4] F. Chen, Q. Xu, and J. S. Hesthaven, A multi-domain spectral method for time-fractional differential equations, J. Comput. Phys., 293(2015), pp. 157-172.

[5] S. Chen, J. Shen, and L.-L. Wang, Generalized Jacobi functions and their applications to fractional differential equations, Math. Comp., 85(2016), pp. 1603-1638.

[6] X. Chen, F. Zeng, and G.E.Karniadakis, A tunable finite difference method for fractional differential equations with non-smooth solutions, Comput. Methods Appl. Mech. Engrg., 318(2017), pp. 193-214.

[7] E. Cuesta, C. Lubich, and C. Palencia, Convolution quadrature time discretization of fractional diffusion-wave equations, Math. Comp., 75(2006), pp. 673- 696.

[8] W. Deng and J. S. Hesthaven, Local discontinuous Galerkin methods for fractional ordinary differential equations, BIT, 55(2015), pp. 967-985.

[9] K. Diethelm, An algorithm for the numerical solution of differential equations of fractional order, Electronic Transactions on Numerical Analysis, 5(1997), pp. 1-6.

[10] K. Diethelm, N. J. Ford, and A. D. Freed, Detailed error analysis for a fractional Adams method, Numer. Algorithms, 36(2004), pp. 31-52.

[11] K. Diethelm, J. M. Ford, N. J. Ford, and M. Weilbeer, Pitfalls in fast numerical solution of fractional differential equations, J. Comput. Appl. Math., 186(2006), pp. 482-503.

[12] P. Flajolet, Singularity analysis and asymptotics of Bernoulli sums, Theoret. Comput. Sci., 215(1999), pp. 371-381.

[13] N. J. Ford, K. Pal, and Y. Yan, An algorithm for the numerical solution of space-fractional partial differential equations, Computational Methods in Applied Mathematics, 15(2015), pp. 497-514.

[14] N. J. Ford, J. Xiao, and Y. Yan, Stability of a numerical method for a space-time-fractional telegraph equation, Computational Methods in Applied Mathematics, 12(2012), pp. 1-16.

[15] N. J. Ford, J. Xiao, and Y. Yan, A finite element method for time-fractional partial differential equations, Fract. Calc. Appl. Anal., 14(2011), pp. 454-474.

[16] G.-H. Gao, Z.-Z. Sun, and H.-W. Zhang, A new fractional numerical differentiation formula to approximate the Caputo fractional derivative and its applications, J. Comput. Phys., 259(2014), pp. 33-50.

[17] R. Gorenflo and F. Mainardi, Random walk models for space fractional diffusion processes, Fract. Calc. Appl. Anal., 1(1998), pp. 167-191. 
[18] Y. Hatano and N. Hatano, Dispersive transport of ions in column experiments: An explanation of long-tailed profiles, Water Res. Research, 34(1998), pp. 1027-1033.

[19] B. Jin, R. Lazarov, and Z. Zhou, Two fully discrete schemes for fractional diffusion and diffusion-wave equations with nonsmooth data, SIAM J. Sci. Comput., 38(2016), pp. A146A170.

[20] B. Jin, R. Lazarov, and Z. Zhou, An analysis of the L1 scheme for the subdiffusion equation with nonsmooth data, IMA J. of Numer. Anal., 36(2016), pp. 197-221.

[21] B. Jin, B. Li, and Z. Zhou, An analysis of the Crank-Nicolson method for subdiffusion, IMA J. of Numer. Anal., in press, 2017, arXiv:1607.06948.

[22] B. Jin, B. Li, and Z. Zhou, Correction of high-order BDF convolution quadrature for fractional evolution equations, preprint, arXiv:1703.08808, 2017.

[23] Z. Li, Z. Liang, and Y. Yan, High-order numerical methods for solving time fractional partial differential equations, J. Sci. Comput., 71(2017), pp. 785-803.

[24] C. Li and H. Ding, Higher order finite difference method for the reaction and anomalousdiffusion equation, Appl. Math. Model., 38 (2014), pp. 3802-3821.

[25] Y. Lin and C. Xu, Finite difference/spectral approximations for the time-fractional diffusion equation, J. Comput. Phys., 225(2007), pp. 1533-1552.

[26] C. Lv and C. Xu, Error analysis of a high order method for time-fractional diffusion equations, SIAM J. Sci. Comput., 38(2016), pp. A2699-A2724.

[27] C. Lubich, Discretized fractional calculus, SIAM J. Math. Anal., 17(1986), pp. 704-719.

[28] C. Lubich, Convolution quadrature and discretized operational calculus. I., Numer. Math., 52(1988), pp. 129-145.

[29] C. Lubich, Convolution quadrature revisited, BIT, 44(2004), pp. 503-514.

[30] C. Lubich, I. H. Sloan, and V. Thomée, Nonsmooth data error estimates for approximations of an evolution equation with a positive-type memory term, Math. Comp., 65(1996), pp. $1-17$.

[31] R. Metzler and J. Klafter, The restaurant at the end of the random walk: Recent developments in the description of anomalous transport by fractional dynamics, J. Phys. A: Math. Gen., 37(2004), pp. 161-208.

[32] K. Mustapha, Time-stepping discontinuous Galerkin methods for fractional diffusion problems, Numer. Math., 130(2015), pp. 497-516.

[33] K. Mustapha, B. Abdallah, and K. M. Furati, A discontinuous Petrov-Galerkin method for time-fractional diffusion equations, SIAM J. Numer. Anal., 52(2014), pp. 2512-2529.

[34] K. Mustapha and D. Schötzau, Well-posedness of hp-version discontinuous Galerkin methods for fractional diffusion wave equations, IMA J. Numer. Anal., 34(2014), pp. 1426-1446.

[35] R. Nigmatulin, The realization of the generalized transfer equation in a medium with fractal geometry, Phys. Stat. Sol. B, 133(1986), pp. 425-430.

[36] J. M. Sanz-Serna, A numerical method for a partial integral-differential equation, SIAM J. Numer. Anal., 25(1988), pp. 319-327, 2011.

[37] Z.-Z. Sun and X. Wu, A fully discrete scheme for a diffusion wave system, Appl. Numer. Math., 56(2011), pp. 193-209.

[38] V. Thomée, Galerkin Finite Element Methods for Parabolic Problems, Springer-Verlag, Berlin, 2007.

[39] M. Weilbeer, Efficient Numerical Methods for Fractional Differential Equations and their Analytical Background, Dissertation, Technischen Universitat Braunschweig, 2005.

[40] R. Wu, H. Ding and C. Li, Determination of coefficients of high-order schemes for RiemannLiouville derivative, The Scientific World Journal, 2014, 2014.

[41] Y. Yan, K. Pal, and N. J. Ford, Higher order numerical methods for solving fractional differential equations, BIT Numer Math, 54(2014), pp. 555-584.

[42] Y. Yu, P. Perdikaris, and G.E. Karniadakis, Fractional modeling of viscoelasticity in $3 d$ cerebral arteries and aneurysms, J. Comput. Phys., 323(2016), pp. 219-242.

[43] M. Zayernouri, M. Ainsworth, and G. E. Karniadakis, A unified Petrov-Galerkin spectral method for fractional pdes, Comput. Methods Appl. Mech. Engrg., 283(2015), pp. 15451569.

[44] M. Zayernouri and A. Matzavinos, Fractional Adams-Bashforth/Moulton methods: An application to the fractional Keller-Segel chemotaxis system, J. Comput. Phys., 317(2016), pp. $1-14$.

[45] M. Zayernouri and G. E. Karniadakis, Fractional spectral collocation method, SIAM J. Sci. Comput., 36(2014), pp. A40-A62.

[46] F. Zeng, C. Li, F. Liu, and I. Turner, The use of finite difference/element approaches for solving the time-fractional subdiffusion equation, SIAM J. Sci. Comput., 35(2013), pp. A2976-A3000. 
[47] F. Zeng, Z. Zhang, and G. E. Karniadakis, Second-order numerical methods for multi-term fractional differential equations: Smooth and non-smooth solutions, arXiv: 1701.00996, 2017. 